\title{
A VARIABLE MID-INFRARED SYNCHROTRON BREAK ASSOCIATED WITH THE COMPACT JET IN GX 339-4
}

\author{
P. Gandhi ${ }^{1}$, A. W. Blain ${ }^{2}$, D. M. Russell ${ }^{3}$, P. Casella ${ }^{4}$, J. MalzaC $^{5,6}$, S. Corbel $^{7}$, P. D' Avanzo ${ }^{8}$, F. W. Lewis $^{9}$, \\ S. MarkofF ${ }^{3}$, M. Cadolle Bel ${ }^{10}$, P. Goldoni $^{11,12}$, S. Wachter ${ }^{13}$, D. Khangulyan ${ }^{1}$, And A. MainzeR ${ }^{14}$ \\ ${ }^{1}$ Institute of Space and Astronautical Science, Japan Aerospace Exploration Agency, 3-1-1 Yoshinodai, chuo-ku, Sagamihara, Kanagawa 252-5210, Japan \\ ${ }^{2}$ Department of Physics \& Astronomy, University of Leicester, University Road, Leicester LE1 7RH, UK \\ ${ }^{3}$ Astronomical Institute “Anton Pannekoek," University of Amsterdam, P.O. Box 94249, 1090 GE Amsterdam, The Netherlands \\ ${ }^{4}$ School of Physics and Astronomy, University of Southampton, Southampton SO17 1BJ, UK \\ ${ }_{5}^{5}$ Université de Toulouse, UPS-OMP, IRAP, Toulouse, France \\ ${ }^{6}$ CNRS, IRAP, 9 Av. colonel Roche, BP 44346, F-31028 Toulouse cedex 4, France \\ ${ }^{7}$ Université Paris 7 Denis Diderot and Service d'Astrophysique, UMR AIM, CEA Saclay, F-91191 Gif sur Yvette, France \\ ${ }^{8}$ INAF-Osservatorio Astronomico di Brera, via Bianchi 46, I-23807, Merate (Lc), Italy \\ ${ }^{9}$ Faulkes Telescope Project, Division of Earth, Space and Environment, University of Glamorgan, Pontypridd CF37 1DL, UK \\ ${ }^{10}$ INTEGRAL Science Operations Centre Science Operations Department, European Space Astronomy Centre, Post Office Box 78, E-28691, \\ Villanuevade la Caada, Madrid, Spain \\ ${ }^{11}$ Laboratoire Astroparticule et Cosmologie, 10 rue A. Domon et L. Duquet, 75205 Paris Cedex 13, France \\ ${ }^{12}$ DSM/IRFU/Service d'Astrophysique, CEA/Saclay, 91191 Gif-sur-Yvette, France \\ ${ }^{13}$ Infrared Processing and Analysis Center, California Institute of Technology, Pasadena, CA 91125, USA \\ 14 Jet Propulsion Laboratory, California Institute of Technology, Pasadena, CA 91109, USA \\ Received 2011 August 5; accepted 2011 August 25; published 2011 September 20
}

\begin{abstract}
Many X-ray binaries remain undetected in the mid-infrared, a regime where emission from their compact jets is likely to dominate. Here, we report the detection of the black hole binary GX 339-4 with the Wide-field Infrared Survey Explorer (WISE) during a very bright, hard accretion state in 2010. Combined with a rich contemporaneous multiwavelength data set, clear spectral curvature is found in the infrared, associated with the peak flux density expected from the compact jet. An optically thin slope of $\sim-0.7$ and a jet radiative power of $>6 \times 10^{35} \mathrm{erg} \mathrm{s}^{-1}$ $(d / 8 \mathrm{kpc})^{2}$ are measured. A $\sim 24 \mathrm{hr}$ WISE light curve shows dramatic variations in mid-infrared spectral slope on timescales at least as short as the satellite orbital period $\sim 95$ minutes. There is also significant change during one pair of observations spaced by only $11 \mathrm{~s}$. These variations imply that the spectral break associated with the transition from self-absorbed to optically thin jet synchrotron radiation must be varying across the full wavelength range of $\sim 3-22 \mu \mathrm{m}$ that WISE is sensitive to, and more. Based on four-band simultaneous mid-infrared detections, the break is constrained to frequencies of $\approx 4.6_{-2.0}^{+3.5} \times 10^{13} \mathrm{~Hz}$ in at least two epochs of observation, consistent with a magnetic field $B \approx 1.5( \pm 0.8) \times 10^{4} \mathrm{G}$ assuming a single-zone synchrotron emission region. The observed variability implies that either $B$ or the size of the acceleration zone above the jet base is being modulated by factors of $\sim 10$ on relatively short timescales.
\end{abstract}

Key words: accretion, accretion disks - infrared: general - radiation mechanisms: non-thermal - stars: individual (GX339-4) - X-rays: binaries

\section{INTRODUCTION}

Despite decades of studies on accreting sources, observations of jets in X-ray binaries suffer from important limitations. Strong emission from accreting, stellar, or surrounding material dominates fainter jet radiation over infrared-X-ray frequencies in most sources (e.g., Russell et al. 2006). The transient nature of activity in many binaries makes it difficult to catch them contemporaneously with multiple observatories necessary to probe broadband emission and isolate the jet. Moreover, there has so far been a lack of sensitive monitoring instruments in the infrared where the jet flux density is expected to peak (e.g., Markoff et al. 2001).

According to the canonical model of emission from compact jets (Blandford \& Königl 1979), this peak occurs because conservation of particle and magnetic flux leads to each location in the jet being associated with its own photospheric frequency, which falls linearly with distance from the base. The sum of all components conspires to give a flat/inverted spectrum $\left(\alpha \geqslant 0\right.$, where $\left.F_{v} \propto v^{\alpha}\right)$ up to a peak frequency associated with the optically thick-to-thin break $\left(v_{\mathrm{b}}\right)$, above which optically thin synchrotron radiation $(\alpha<0)$ reveals information about the underlying particle distribution. Constraints on this break remain sparse due to the limitations mentioned above (e.g., Fender 2001; Corbel \& Fender 2002; Gallo et al. 2007; Migliari et al. 2007, 2010; Pe'er \& Casella 2009; Rahoui et al. 2011).

In this Letter, we present the first mid-infrared (MIR) study of GX 339-4, a binary hosting a black hole with mass $\gtrsim 6 M_{\odot}$ (Hynes et al. 2003) and well known for its transient, multiwavelength variability on a broad range of timescales (Motch et al. 1982; Dunn et al. 2008; Gandhi 2009; Casella et al. 2010). Its donor star is a late-type companion in a long $(\sim 1.7$ days $)$ orbit, much fainter than the primary when active (Shahbaz et al. 2001; Muñoz-Darias et al. 2008). GX 339-4 shows evidence of relativistic jets and has proven to be a key object for jet studies across the electromagnetic spectrum (Fender 2001; Corbel et al. 2003; Markoff et al. 2003; Gandhi et al. 2008).

A MIR detection with Spitzer has been reported, but only in a single filter during a faint, steep-power-law state (Tomsick et al. 2004). We now present multiband simultaneous MIR detections during the source outburst of 2010, which allow important new constraints on $v_{\mathrm{b}}$ and on changing conditions in the inner jet. A distance of $8 \mathrm{kpc}$ and a black hole mass of $10 M_{\odot}$ are taken as representative (Shidatsu et al. 2011). Interstellar line-of-sight extinction of $A_{V}=3.25$ is assumed (F. W. Lewis et al. 2012, in preparation), with dereddening uncertainties described in detail. 
Table 1

GX 339-4 WISE Photometry

\begin{tabular}{|c|c|c|c|c|c|c|c|}
\hline Epoch & MJD & $\begin{array}{c}\mathrm{W} 1^{\mathrm{a}} \\
(\mathrm{mag})(\mathrm{mJy})\end{array}$ & $\begin{array}{c}\text { W2 } 2^{\mathrm{a}} \\
(\mathrm{mag})(\mathrm{mJy})\end{array}$ & $\begin{array}{c}\mathrm{W}^{\mathrm{a}} \\
(\mathrm{mag})(\mathrm{mJy})\end{array}$ & $\begin{array}{c}\text { W4 }{ }^{\mathrm{a}} \\
(\mathrm{mag})(\mathrm{mJy})\end{array}$ & $\alpha_{\text {WISE }}^{\mathrm{b}}$ & $\begin{array}{l}P^{\mathrm{c}} \\
(\%)\end{array}$ \\
\hline 1 & 55265.8849 & $9.96 \pm 0.03(39.4 \pm 2)$ & $9.19 \pm 0.02(42.3 \pm 2)$ & $7.57 \pm 0.04(36.5 \pm 3)$ & $5.86 \pm 0.11(44.1 \pm 7)$ & +0.02 & 82 \\
\hline 2 & 55266.0173 & $9.31 \pm 0.02(71.7 \pm 3)$ & $8.54 \pm 0.02(77.3 \pm 3)$ & $6.86 \pm 0.03(70.1 \pm 5)$ & $5.57 \pm 0.07(57.5 \pm 7)$ & +0.09 & 86 \\
\hline 3 & 55266.1496 & $9.51 \pm 0.03(59.5 \pm 3)$ & $8.58 \pm 0.02(74.2 \pm 3)$ & $6.37 \pm 0.02(109.6 \pm 8)$ & $4.79 \pm 0.04(118.8 \pm 12)$ & -0.40 & 88 \\
\hline 4 & 55266.1497 & $9.83 \pm 0.03(44.4 \pm 2)$ & $8.87 \pm 0.03(57.2 \pm 3)$ & $6.64 \pm 0.02(85.8 \pm 6)$ & $4.81 \pm 0.04(116.2 \pm 11)$ & -0.50 & 66 \\
\hline 5 & 55266.2158 & $9.45 \pm 0.02(63.3 \pm 3)$ & $8.63 \pm 0.02(70.8 \pm 3)$ & $6.45 \pm 0.03(102.1 \pm 7)$ & $4.86 \pm 0.03(110.6 \pm 11)$ & -0.33 & 48 \\
\hline 6 & 55266.2820 & $9.95 \pm 0.02(39.9 \pm 2)$ & $8.99 \pm 0.02(50.8 \pm 2)$ & $6.76 \pm 0.03(76.5 \pm 6)$ & $5.12 \pm 0.09(87.1 \pm 11)$ & -0.46 & 85 \\
\hline 7 & 55266.3481 & $9.78 \pm 0.02(46.6 \pm 2)$ & $8.96 \pm 0.03(52.5 \pm 2)$ & $7.27 \pm 0.04(47.9 \pm 4)$ & $5.71 \pm 0.06(50.9 \pm 5)$ & -0.02 & 82 \\
\hline 8 & 55266.4143 & $9.72 \pm 0.03(49.4 \pm 2)$ & $9.01 \pm 0.02(49.9 \pm 2)$ & $7.43 \pm 0.03(41.4 \pm 3)$ & $6.07 \pm 0.09(36.5 \pm 5)$ & +0.16 & 40 \\
\hline 9 & 55266.4804 & $9.61 \pm 0.03(54.6 \pm 3)$ & $8.73 \pm 0.03(64.6 \pm 3)$ & $6.67 \pm 0.03(83.7 \pm 6)$ & $5.05 \pm 0.06(93.6 \pm 10)$ & -0.30 & 56 \\
\hline 10 & 55266.5466 & $9.35 \pm 0.02(69.1 \pm 3)$ & $8.55 \pm 0.01(76.3 \pm 3)$ & $6.59 \pm 0.02(89.9 \pm 6)$ & $5.00 \pm 0.04(97.3 \pm 10)$ & -0.19 & 25 \\
\hline 11 & 55266.6128 & $10.09 \pm 0.03(35.1 \pm 2)$ & $9.18 \pm 0.02(43.0 \pm 2)$ & $6.88 \pm 0.03(68.9 \pm 5)$ & $5.05 \pm 0.07(93.5 \pm 10)$ & -0.52 & 17 \\
\hline 12 & 55266.7451 & $9.45 \pm 0.03(63.1 \pm 3)$ & $8.51 \pm 0.02(79.3 \pm 3)$ & $6.37 \pm 0.02(109.6 \pm 8)$ & $4.81 \pm 0.04(116.1 \pm 11)$ & -0.35 & 93 \\
\hline 13 & 55266.8774 & $9.18 \pm 0.03(80.9 \pm 4)$ & $8.28 \pm 0.03(98.1 \pm 5)$ & $6.30 \pm 0.02(116.7 \pm 8)$ & $4.83 \pm 0.04(114.6 \pm 11)$ & -0.21 & 93 \\
\hline
\end{tabular}

Notes.

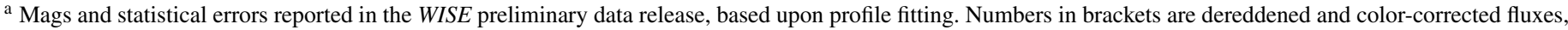
with errors including systematics.

b Spectral slope from single-power-law fit to each flux-calibrated WISE epoch. Uncertainties are $\approx 0.04-0.05(1 \sigma)$.

${ }^{\mathrm{c}}$ Null hypothesis probability at which a single-power-law fit may be rejected, based upon $\chi^{2}$ statistics with two degrees of freedom.

\section{OBSERVATIONS}

\subsection{WISE}

In the course of an all-sky survey, the Wide-field Infrared Survey Explorer (WISE) satellite (Wright et al. 2010, hereafter W10) detected GX 339-4 on 2010 March 11 (MJD 55266). This fortuitously occurred during a bright, hard state phase before outburst peak in April (e.g., Belloni et al. 2010).

WISE has four arrays (bands W1-W4 centered on wavelengths of 3.4, 4.6, 12, and $22 \mu \mathrm{m}$, respectively) which image the same $47^{\prime} \times 47^{\prime}$ field of view simultaneously using three dichroic beam splitters. The frame exposure times are $7.7 \mathrm{~s}$ for W1-2, and $8.8 \mathrm{~s}$ for W3-4, respectively. Consecutive frames are separated by $11 \mathrm{~s}$ as the satellite scans across the sky. Though not designed for timing studies, the surveying strategy was such that every source was scanned multiple times over a period of $\sim 24 \mathrm{hr}$, sampling variability on timescales as short as the satellite orbital period. There is a $\sim 10 \%$ field overlap between adjacent scans, thus sources may be caught on occasion only $11 \mathrm{~s}$ apart.

The WISE all-sky survey preliminary data release ${ }^{15}$ reports Vega source magnitudes using both profile fitting and standard aperture-corrected photometry. Profile fitting represents the most accurate flux estimation for point sources for single-frame measurements ${ }^{16}$ because the pipeline can account for image artifacts and source confusion. Some bad pixels are present around GX 339-4 but the field is free of diffraction spikes and detector latency caused by saturated bright sources. The pointspread function corresponds to a Gaussian of about 6.'1-6.'5 (W1-W3) and 12" (W4), respectively, imaged at 2".8 pixel $^{-1}$ (W1-W3) and 5".6 pixel $^{-1}$ (W4). The two nearest bright stars to GX 339-4 lie $\sim 9^{\prime \prime}$ and $14^{\prime \prime}$ away, with mean central-pixel count rates of $\sim 0.1$ and 1.5 times GX 339-4 in W1 (and a decreasing count rate toward W4). The pipeline successfully deblends multicomponent emission and reports acceptable $\chi^{2}$ values for the photometric fits.

WISE absolute calibration is referenced to a network of standard stars (Cohen et al. 1999) and to sources at the

\footnotetext{
15 http://wise2.ipac.caltech.edu/docs/release/prelim/

$16 \mathrm{http}: / /$ wise2.ipac.caltech.edu/docs/release/prelim/expsup/sec4_3c.htm
}

ecliptic poles that are observed on most orbits. GX 339-4 magnitudes are listed in Table 1 for all 13 observing epochs. These were converted to fluxes using standard zero points and color corrections (W10) assuming a flat power law. To account for present pipeline and absolute calibration uncertainties, we include a systematic zero point uncertainty of $2.4 \%, 2.8 \%$, 4.5\%, and 5.7\% in W1-W4, respectively (Jarrett et al. 2011). The color correction mainly affects the W3 band, and we also include a $2 \%$ uncertainty for this (W10), given the range of MIR spectral slopes discussed later. Improved calibration and reduced systematic errors may be expected after the full WISE data release in 2012.

\subsection{Multiwavelength}

Follow-up data will be presented in a number of upcoming papers, including (1) Cadolle Bel et al. (2011, hereafter CB11) who describe extensive X-ray, near-IR, and optical coverage; (2) F. W. Lewis et al. (2012, in preparation) presenting further optical and UV data; and (3) S. Corbel et al. (2012, in preparation) discussing the radio. Broadband monochromatic fluxes closest to the WISE observations are listed in Table 2 and plotted in Figure 1. Where multiple observations were available, averages were used for the mean spectral energy distribution (SED), with the mean error computed as the standard deviation divided by the square root of the number of exposures.

Below we present some details on the X-ray analysis, the radio observations, and the UV-to-MIR dereddening, which are specific to our work.

\subsubsection{X-Rays}

Public X-ray data nearly simultaneous with WISE from the Rossi X-ray Timing Explorer (RXTE; Bradt et al. 1993) were available for analysis. A short observation (ObsId 95409-01$09-03$ ) with goodtime $\approx 1360 \mathrm{~s}$ was conducted between WISE epochs 12 and 13. For data reduction, we followed standard procedures using HEASOFT v.6.10 and FTOOLS (similarly to Gandhi et al. 2010, hereafter G10).

We find an acceptable fit with a multicolor disk with inner temperature $k T_{\text {in }}=0.80_{-0.25}^{+0.15} \mathrm{keV}(90 \%$ confidence 
Table 2

Quasi-simultaneous Dereddened Ultraviolet to Radio Mean Fluxes

\begin{tabular}{|c|c|c|c|c|}
\hline Band (Telescope or Instrument) ${ }^{\mathrm{a}}$ & $\begin{array}{c}\log \text { Frequency } \\
(\mathrm{Hz})\end{array}$ & Mean $\mathrm{MJD}^{\mathrm{b}}$ & $\begin{array}{l}\text { Flux Density } \\
\qquad(\mathrm{mJy})\end{array}$ & References \\
\hline$U V W 2$ (UVOT) & 15.19 & 55261.05 & $83.8( \pm 6.9)_{-59}^{+203}$ & CB11; F. W. Lewis et al. (2012, in preparation) \\
\hline$U(\mathrm{UVOT})$ & 14.94 & 55260.38 & $40.3( \pm 0.7)_{-21}^{+44}$ & CB11; F. W. Lewis et al. (2012, in preparation) \\
\hline$V(\mathrm{FTS})$ & 14.74 & 55273.72 & $54.5( \pm 1.8)_{-20}^{+32}$ & CB11; F. W. Lewis et al. (2012, in preparation) \\
\hline$R(\mathrm{FTS})$ & 14.67 & 55273.72 & $33.2( \pm 2.1)_{-10}^{+14}$ & CB11; F. W. Lewis et al. (2012, in preparation) \\
\hline$i^{\prime}(\mathrm{FTS})$ & 14.60 & 55273.72 & $33.9( \pm 0.3)_{-9}^{+12}$ & CB11; F. W. Lewis et al. (2012, in preparation) \\
\hline$V(\mathrm{REM})$ & 14.74 & 55261.30 & $48.2( \pm 1.1)_{-18}^{+28}$ & CB11 \\
\hline$R(\mathrm{REM})$ & 14.67 & 55261.30 & $30.7( \pm 0.7)_{-9}^{+13}$ & CB11 \\
\hline$I(\mathrm{REM})$ & 14.58 & 55261.30 & $29.9( \pm 0.3)_{-6}^{+7}$ & CB11 \\
\hline$J(\mathrm{REM})$ & 14.39 & 55261.30 & $32.4( \pm 0.8)_{-4}^{+5}$ & CB11 \\
\hline$H(\mathrm{REM})$ & 14.26 & 55261.30 & $35.1( \pm 0.3)_{-3}^{+3}$ & CB11 \\
\hline W1 (WISE) & 13.95 & 55266.363 & $55.2( \pm 3.9)_{-4}^{+5}$ & This work \\
\hline W2 (WISE) & 13.81 & 55266.363 & $64.3( \pm 4.6)_{-5}^{+5}$ & This work \\
\hline W3 (WISE) & 13.41 & 55266.363 & $79.9( \pm 7.3)_{-9}^{+10}$ & This work \\
\hline W4 (WISE) & 13.13 & 55266.363 & $87.4( \pm 8.3)_{-11}^{+12}$ & This work \\
\hline $9 \mathrm{GHz}$ (ATCA) & 9.95 & 55266.4 & $9.7( \pm 0.1) \pm 1.7$ & S. Corbel et al. (2012, in preparation) \\
\hline $5.5 \mathrm{GHz}(\mathrm{ATCA})$ & 9.74 & 55266.4 & $9.1( \pm 0.1) \pm 1.0$ & S. Corbel et al. (2012, in preparation) \\
\hline
\end{tabular}

Notes.

${ }^{a}$ UVOT: Swift UltraViolet and Optical Telescope; FTS: Faulkes Telescope South; REM: Rapid Eye Mount telescope.

${ }^{\mathrm{b}}$ Closest available data to the WISE observation.

${ }^{\mathrm{c}}$ Dereddened fluxes. Statistical error bars are in brackets; the following numbers are the total errors including systematics.

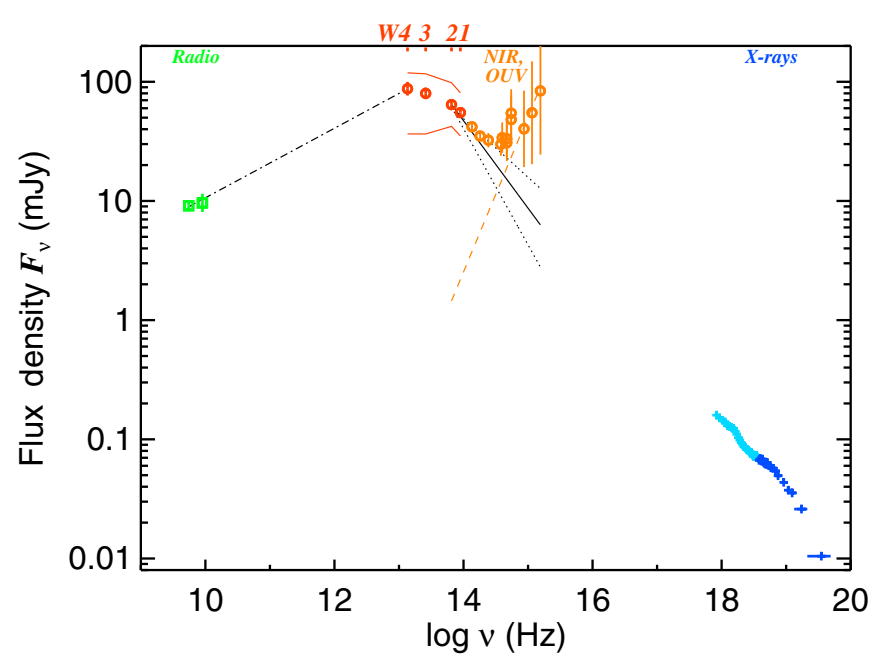

Figure 1. Average dereddened SED of GX 339-4. WISE data points are in red; red curves represent the envelope of extreme variations over 13 WISE epochs. The near-infrared and optical/ultraviolet (OUV) points are plotted in orange and radio in green. The optically thin jet power law is shown as the solid black line, and $1 \sigma$ fit uncertainties on this slope by the dotted lines. The dashed and dotdashed lines represent the OUV and optically thick power laws, respectively. Detailed modeling of the origin of X-rays (blue; unfolded best-fit model) is beyond the scope of this Letter.

intervals), an $\mathrm{Fe} \mathrm{K}$ line with equivalent width ranging over $\sim 70-450 \mathrm{eV}$, and coronal Comptonization with electron temperature $k T_{e}=32_{-3}^{+4} \mathrm{keV}$ and optical depth $\tau=1.87_{-0.20}^{+0.17}$ (modeled with COMPTT; Titarchuk 1994). The Proportional Counter Array (PCA) and HEXTE fluxes are found to be $F_{3-20 \mathrm{keV}}=$ $3.70_{-0.20}^{+0.01} \times 10^{-9}$ and $F_{20-200 \mathrm{keV}}=8.6_{-0.5}^{+0.1} \times 10^{-9} \mathrm{erg} \mathrm{s}^{-1} \mathrm{~cm}^{-2}$, respectively, giving a luminosity $L_{1-200 \mathrm{keV}}=1.0 \times 10^{38} \mathrm{erg} \mathrm{s}^{-1}$ or $\approx 0.08 \times L_{\text {Eddington. }}$ The bright-state spectrum of GX 339-4 is known to be complex and the exact physical model is irrelevant for the work presented here. The derived luminosity is not overly sensitive to model parameterization. See CB11 for detailed X-ray evolution of the source over the outburst.

For timing analysis, we extracted net light curves from the RXTE event mode data, with $8 \mathrm{~s}$ time bins in order to approximately match the WISE exposure times. RXTE data probe variability shorter than $1360 \mathrm{~s}$. For longer timescales, we used Swift Burst Alert Telescope (BAT) 15-50 keV light curves provided by the Hard X-ray Transient Monitor team. ${ }^{17}$ Swift sampling times are non-uniform, with intervals ranging from $\sim 10$ minutes to $\gtrsim 1 \mathrm{hr}$.

\subsubsection{Radio}

The Australia Telescope Compact Array (ATCA) observed GX 339-4 frequently during the outburst. Here, we have used the mean fluxes observed on the two closest dates straddling the WISE observation: MJD 55262.91 and 55269.80, with simultaneous observations in the 5.5 and $9 \mathrm{GHz}$ bands. Variations of $\approx 25 \%$ and $40 \%$ are present between the bands. Note that the source does not display extreme radio variability on timescales of days (Corbel et al. 2000). Full details will be presented in S. Corbel et al. (2012, in preparation).

\subsubsection{Dereddening}

The UV-to-near-IR data were dereddened assuming a standard interstellar extinction law with $R_{V}=3.1$ (Cardelli et al. 1989). Extinction beyond $3 \mu \mathrm{m}$, though certainly lower than in the near-IR, remains ill-constrained. We use the mean of three recent extinction laws for the WISE data, with their dispersion

\footnotetext{
17 http://swift.gsfc.nasa.gov/docs/swift/results/transients/weak/GX339-4
} 
serving as a propagated systematic uncertainty on the corrected fluxes: (1) the $R_{V}=5.5$ grain model of Draine (2003) which matches the Galactic center extinction of Lutz et al. (1996) below $8 \mu \mathrm{m}$; (2) the Galactic center extinction curve described by Chiar \& Tielens (2006); and (3) that presented by Flaherty et al. (2007). The latter two provide updates on MIR silicate absorption strengths. Finally, we also include a systematic $A_{V}$ uncertainty of $0.5 \mathrm{mag}(\mathrm{G} 10)$.

\section{RESULTS}

\subsection{Broadband Spectrum}

GX 339-4 is detected with high significance in all four WISE bands, with average signal-to-noise ratio of $\sim 40-50$ in W1-W3 and 22 in W4 across epochs. With mean observed fluxes of $\gtrsim 60 \mathrm{mJy}$ in $\mathrm{W} 3$ and W4, these detections are among the brightest for transient binary systems in the MIR (Wachter 2008; Vrtilek 2008), especially when considering the isolated jet component, as we discuss below.

The SED in Figure 1 shows two peaks. A rising spectrum in the optical/ultraviolet (OUV) may originate either via preshock jet synchrotron or accretion disk (re)radiation (cf. G10). Dominant dereddening uncertainty prevents detailed inferences here, but we note that the optical-to-X-ray flux ratio is relatively low at $\lambda F_{\lambda}(5500 \AA) / F_{2-10} \sim 0.08$ (with a factor of two uncertainty), so disk $\mathrm{X}$-ray irradiation could provide the requisite power.

A second peak is in the MIR, associated with broad spectral curvature declining toward the near-IR and to the radio. We discuss below that this curvature is likely to be emission from the compact jet, with the WISE W1-2 bands preferentially probing optically thin radiation. Thus fitting a two-power-law model (one for the optically thin jet and one for the OUV) over the W2-UV frequency range yields a mean optically thin spectral slope $\alpha_{\text {thin }}=-0.73 \pm 0.24$, and a largely unconstrained OUV slope $\alpha_{\text {OUV }}=+1.3 \pm 0.9$. Fitting a single optically thick power law to the radio-W4 regime yields $\alpha_{\text {thick }}=+0.29 \pm 0.02$, though the average SED indicates that a single power law may be an oversimplification. Errors include systematic uncertainties.

$\alpha_{\text {thin }}$ measures the distribution function of jet plasma energies, implying a power-law slope $p=1-2 \alpha_{\text {thin }}=2.5 \pm$ 0.5 (Rybicki \& Lightman 1979). Integrating over the mean radio-near-IR $(2 \mu \mathrm{m})$ SED using piece-wise power-law interpolation gives a lower limit to the jet radiative power $\left(L_{\mathrm{rad}}\right)$ of $6 \times 10^{35} \mathrm{erg} \mathrm{s}^{-1}$, assuming that corrections due to relativistic beaming are of order unity (Casella et al. 2010). If the X-ray power $\left(L_{1-200 \mathrm{keV}}\right)$ is close to the total jet power (Körding et al. 2006), we may place a lower limit on the jet radiative efficiency, $\eta=L_{\mathrm{rad}} / L_{\mathrm{jet}} \gtrsim 1 \%$.

\subsection{Variability}

Simultaneous four-band WISE light curves in Figure 2 show striking variability typically sampled at (multiples of) the WISE orbital period of 95 minutes. The shortest sampling interval was $11 \mathrm{~s}$ between epochs 3 and 4, when WISE caught GX 339-4 on two consecutive scans. We extracted light curves of randomly selected field stars for comparison. For each band, two field stars with brightness similar to GX 339-4 within 5' were selected. Variations in the target light curves are clearly much stronger than the field star variations and also display inter-band correlations, implying that they are not related to any instrumental or observational artifact.

We measured the fractional variability amplitudes $\left(F_{\text {var }}\right.$; Vaughan et al. 2003) of the MIR light curves and found them to increase with wavelength, with $F_{\text {var }}^{\mathrm{MIR}} \sim 0.25 \pm 0.03$ for W1-2 and $\sim 0.32 \pm 0.06$ for W3-4, respectively (errors allow for WISE pipeline systematics as in Section 2.1). The peak-to-peak flux ratios also increase from 2.3 to 3.3 in W1-4. In X-rays, the Swift data show low mean variability over the timespan of WISE observations, with $F_{\text {var }}($ Swift $)=0.17 \pm 0.02$. This value may suffer from large systematics because BAT employs a coded mask with a wide field of view. RXTE is not affected by such complications, and we measure $F_{\text {var }}(\mathrm{PCA})=0.21 \pm 0.01$ and $F_{\text {var }}(H E X T E)=0.20 \pm 0.01$ for the full energy ranges of the instruments, and over the range of timescales of 8-1360 s.

\section{DISCUSSION}

Both the timing and spectral properties strongly favor a jet origin for the mid-infrared emission. The mean optically thick and thin slopes are fairly typical for binaries in the hard state when the jet is known to be strong. From closely simultaneous $\mathrm{X}$-ray and MIR light curves, we find $F_{\text {var }}^{\mathrm{X} \text {-ray }}<F_{\text {var }}^{\mathrm{MIR}}$ when comparing similar timescales. This argues against strong MIR variations originating via reprocessing, because the secondary (driven) variability cannot exceed the primary ones in strength. $\mathrm{X}$-ray variations can also be ruled out as the driver of IR variations in the outer disk because the IR spectral slopes are inconsistent with thermal reprocessing. Dusty circumbinary disks found in some other sources (Rahoui et al. 2010) are unlikely here because of the strong variability (so dust grains should be destroyed by X-rays), and the MIR spectral slopes do not match thermal dust emission.

The strong variability implies dramatic changes in spectral slope across the WISE bands in the individual observation epochs. These are displayed in Figure 3. Parameterizing the spectra with single power laws results in slopes of $\alpha_{\text {WISE }} \sim$ -0.5 to +0.2 (Table 1 ). In several epochs, additional spectral curvature appears to be present within the WISE bands. In particular, in epochs 12 and 13, W1 and W4 fluxes lie below the fit (while W2-3 lie above), returning large single-power-law null-hypothesis rejection probabilities $(P)$. From W2 to W1, the slope is negative in most epochs $\left(\overline{\alpha_{W 1}^{W 2}}=-0.48 \pm 0.07\right)$, meaning that these bands probe mostly optically thin emission. Thus carrying out a simple two-power-law fit to describe the $\mathrm{W} 1$ and W2 band pair and the W3 and W4 pair separately, the spectral break associated with this curvature is constrained to be at $\log \left(v_{\mathrm{b}} ; \mathrm{Hz}\right)=13.65 \pm 0.24$ and $13.68 \pm 0.26$ in epochs 12 and 13 , respectively.

The position of $v_{\mathrm{b}}$ depends strongly on the magnetic field strength $(B)$ at the acceleration zone in the compact jet, and is comparatively insensitive to luminosity, geometry, and energy partition factors. Generalizing the homogeneous cylindrical single-zone emission model of Chaty et al. (2011), we find expressions for $B$ and the zone radius $R$ as follows:

$$
\begin{aligned}
B= & 1.5 \times 10^{4} \xi^{-4 z} h^{2 z} d_{8}^{-4 z} \\
& \times\left(F_{v_{\mathrm{b}}} / F_{70}\right)^{-2 z}\left(\nu_{\mathrm{b}} / 4.5 \times 10^{13}\right) \mathrm{G}, \\
R= & 2.2 \times 10^{9} \xi^{-z} h^{-z(p+6)} d_{8}^{2 z(p+6)} \\
& \times\left(F_{v_{\mathrm{b}}} / F_{70}\right)^{z(p+6)}\left(v_{\mathrm{b}} / 4.5 \times 10^{13}\right)^{-1} \mathrm{~cm},
\end{aligned}
$$

where $z=(2 p+13)^{-1}, \xi$ is the equipartition fraction of particles to magnetic energy density, $h$ is the scale height of the optically thin region, $d_{8}$ is the distance in units of $8 \mathrm{kpc}$, and $F_{70}=$ $70 \mathrm{mJy}$. Since $B$ depends only weakly on $h$ and $\xi$ (we fix both 

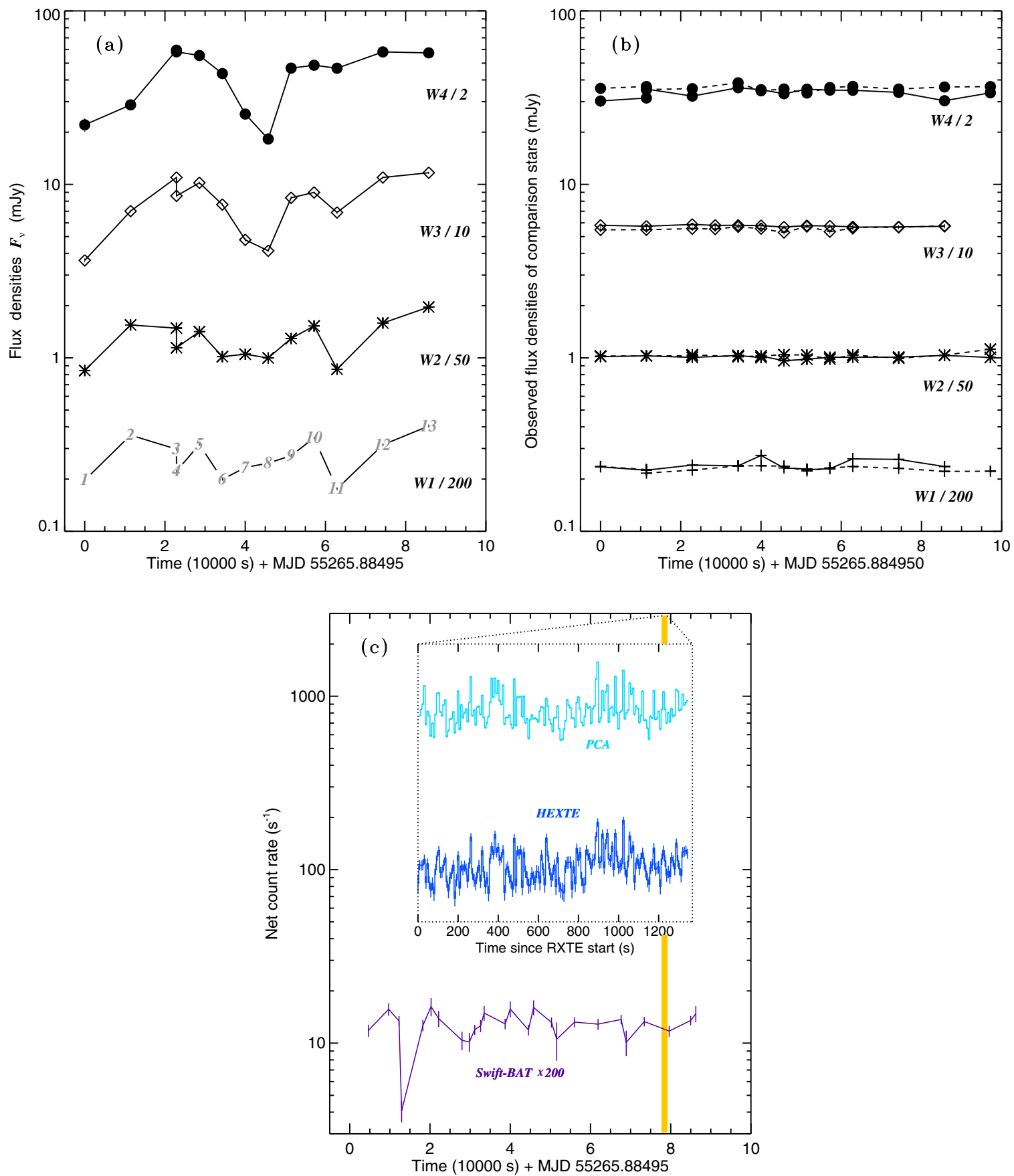

Figure 2. (a) WISE flux light curves of GX 339-4 showing the 13 epochs of measurement. Statistical error bars are typically smaller than the plot symbols. Band light curves have been offset by the labeled factors for clarity. (b) Light curves for randomly selected comparison field stars (two stars per band are shown as the solid and dashed lines, respectively). (c) X-ray light curves. The 15-50 keV Swift/BAT hard X-ray light curve covers a full day. The RXTE observing period is denoted in yellow, with the inset showing the PCA and HEXTE light curves in detail. The $y$-axis has an identical relative scaling in all panels.

at 1), we have a robust estimate of $B$ when $\nu_{\mathrm{b}}$ can be measured. Both epochs 12 and 13 imply $B \approx 1.5( \pm 0.8) \times 10^{4} \mathrm{G}$ and $R \approx$ $2.5( \pm 1.5) \times 10^{9} \mathrm{~cm}$, with errors determined from Monte Carlo sampling of all parameters (including also systematic distance uncertainty of $2 \mathrm{kpc}$ ).

In addition, the strong MIR slope changes between optically thick and thin imply that $\nu_{\mathrm{b}}$ is varying over a range at least as wide as that which WISE is sensitive to. It is likely that uncertainties due to present WISE photometric calibration as well as the ill-constrained dereddening prevent accurate localization of $v_{\mathrm{b}}$ in some other epochs. Setting the WISE systematic errors to zero and repeating the single-power-law fits returns $P>90 \%$ values in additional epochs $1,2,3,6$, and 7, consistent with $v_{\mathrm{b}}$ falling within the WISE bandpass in these.

In order to understand the extent of changing jet physical conditions, we compare two extreme WISE epochs: (1) epoch 4 , with very bright flux in W4 and a steep slope implying optically thin emission over the whole WISE range and (2) epoch 8 , when the source was faintest in W4 with an inverted slope implying that $\nu_{\mathrm{b}}$ has shifted to the high-frequency end. We assume (1) $\nu_{\mathrm{b}}($ epoch 4$)=1.3 \times 10^{13} \mathrm{~Hz}$ and (2) $\nu_{\mathrm{b}}(8)=$ $9 \times 10^{13} \mathrm{~Hz}$, corresponding to the extreme WISE frequencies and representing likely upper and lower limits to the true $\nu_{\mathrm{b}}$ values, respectively. The dereddened fluxes are $F_{\nu_{\mathrm{b}}}(4) \approx$ 


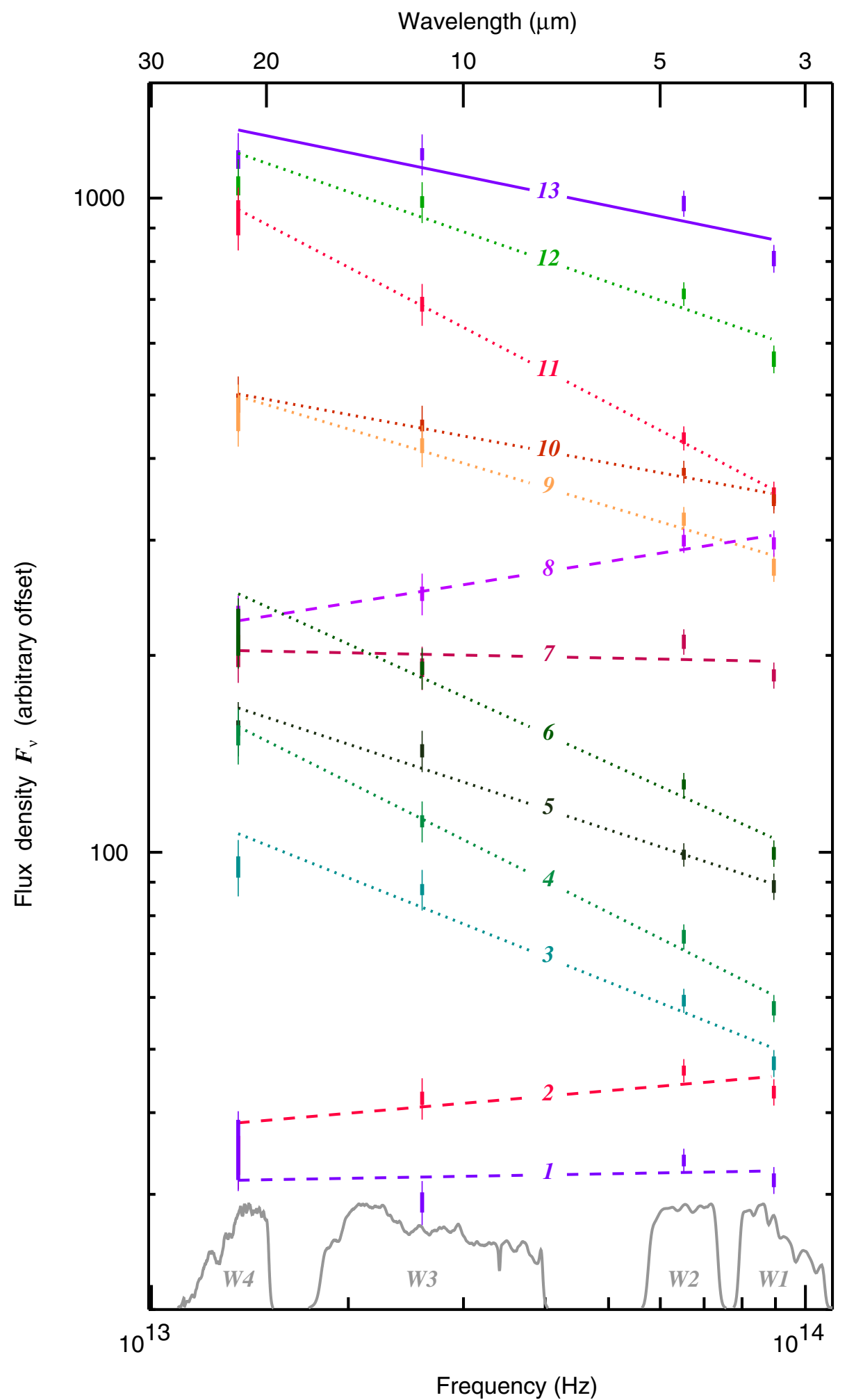

Figure 3. Evolution of the WISE spectra across epochs (labeled) with single-power-law fits overplotted. Epochs 1-13 have multiplicative offsets of 0.8, 0.6, 0.8, 1.3, $1.4,2.5,4,6,5,5,10,9$, and 10, respectively, for clarity. Thick error bars are statistical uncertainties and thin ones include systematic uncertainties. Response profiles of the WISE bands are plotted at the bottom, arbitrarily normalized.

$115 \mathrm{mJy}$ and $F_{v_{\mathrm{b}}}(8) \approx 50 \mathrm{mJy}$. Comparing $B$ and $R$, we find

$$
\begin{gathered}
B(4) / B(8)=\left[F_{\nu_{\mathrm{b}}}(4) / F_{\nu_{\mathrm{b}}}(8)\right]^{-2 z}\left[\nu_{\mathrm{b}}(4) / \nu_{\mathrm{b}}(8)\right]=0.13 \\
R(4) / R(8)=\left[F_{\nu_{\mathrm{b}}}(4) / F_{\nu_{\mathrm{b}}}(8)\right]^{z(p+6)}\left[\nu_{\mathrm{b}}(4) / \nu_{\mathrm{b}}(8)\right]^{-1}=10.4
\end{gathered}
$$

Modulations by factors of $\sim 10$ in the field strength or in acceleration zone size are required. The standard scaling of the $B$ field along the jet is $B \sim R^{-1}$ (Blandford \& Königl 1979) which, if true, is consistent with the variability being dominated by changes in location of the acceleration zone along the jet. These modulations may ultimately originate from stochastic instabilities within the accretion flow, or as a result 
of changing dissipative shock conditions in the inner jet (e.g., Falcke \& Biermann 1995; Meier et al. 2001; Polko et al. 2010). Multiwavelength monitoring coordinated with the mid-infrared opens up the possibility for studying these processes in the future. The characteristic timescales of change are $\sim$ hours or less (Figure 2), though variability between epochs 3 and 4 means that some changes are faster than $\sim 11 \mathrm{~s}$.

Simultaneous multiband observations over a broad frequency range with WISE have proved crucial for revealing complex and rapid changes occurring near the jet base. Based on the average data in Figure 1 alone, it would be impossible to accurately localize $\nu_{\mathrm{b}}$ and to infer changes in its position. Whereas $\nu_{\mathrm{b}}$ has been found to lie at similar MIR frequencies of (1-4) $\times$ $10^{13} \mathrm{~Hz}$ in 4U 0614+091 and Cyg X-1, observational limitations prevented simultaneous observations straddling the break in the former (Migliari et al. 2010), and the MIR is dominated by nonjet emission in the latter (Rahoui et al. 2011). We have cleanly probed jet-dominant MIR emission simultaneously in multiple bands and shown that inferences from long-term averages are not representative of the underlying behavior in GX 339-4 at least. Thus, time dependence must be incorporated as an integral parameter when modeling compact jets.

Scale-invariant jet models imply that similar break frequency variability could be present in active galactic nuclei, where $v_{\mathrm{b}}$ is expected at millimeter frequencies (Kellermann \& Pauliny-Toth 1969; Heinz \& Sunyaev 2003). Long-term millimeter monitoring may have already found signatures for this (Trippe et al. 2011).

WISE is a joint project of the University of California, Los Angeles, and the Jet Propulsion Laboratory (JPL)/California Institute of Technology (Caltech), funded by the National Aeronautics and Space Administration (NASA). Data for individual scans are from NEOWISE (e.g., Mainzer et al. 2011), a project of JPL/Caltech funded by NASA's Planetary Science Division. We thank the referee for a prompt report. Individual support acknowledgments are as follows. P.Ga. acknowledges JAXA International Top Young Fellowship, D.M.R. and S.M. acknowledge Netherlands Organisation for Scientific Research Veni and Vidi Fellowship, respectively, J.M. acknowledges GDR PCHE (France), and P.C. acknowledges EU Marie Curie Intra-European Fellowship 2009-237722. Swift/BAT transient monitor results and RXTE HEASARC archive data are used herein. The Faulkes Telescope South (FTS) is maintained and operated by Las Cumbres Observatory Global Telescope Network. We thank Rosa Doran of NUCLIO, Portugal for FTS observations as part of the EU-Hands on Universe initiative for school scientific education.

\section{REFERENCES}

Belloni, T., Motta, S., \& Muñoz-Darias, T. 2010, ATel, 2577, 1

Blandford, R. D., \& Konigl, A. 1979, ApJ, 232, 34
Bradt, H. V., Rothschild, R. E., \& Swank, J. H. 1993, A\&AS, 97, 355

Cadolle Bel, M., Rodriguez, J., D’Avanzo, P., et al. 2011, A\&A, submitted (CB11)

Cardelli, J. A., Clayton, G. C., \& Mathis, J. S. 1989, ApJ, 345, 245

Casella, P., Maccarone, T. J., O’Brien, K., et al. 2010, MNRAS, 404, L21

Chaty, S., Dubus, G., \& Raichoor, A. 2011, A\&A, 529, A3

Chiar, J. E., \& Tielens, A. G. G. M. 2006, ApJ, 637, 774

Cohen, M., Walker, R. G., Carter, B., et al. 1999, AJ, 117, 1864

Corbel, S., Fender, R. P., Tzioumis, A. K., et al. 2000, A\&A, 359, 251

Corbel, S., \& Fender, R. P. 2002, ApJ, 573, L35

Corbel, S., Nowak, M. A., Fender, R. P., Tzioumis, A. K., \& Markoff, S. 2003, A\&A, 400, 1007

Draine, B. T. 2003, ARA\&A, 41, 241

Dunn, R. J. H., Fender, R. P., Körding, E. G., Cabanac, C., \& Belloni, T. 2008, MNRAS, 387, 545

Falcke, H., \& Biermann, P. L. 1995, A\&A, 293, 665

Fender, R. P. 2001, MNRAS, 322, 31

Flaherty, K. M., Pipher, J. L., Megeath, S. T., et al. 2007, ApJ, 663, 1069

Gallo, E., Migliari, S., Markoff, S., et al. 2007, ApJ, 670, 600

Gandhi, P. 2009, ApJ, 697, L167

Gandhi, P., Dhillon, V. S., Durant, M., et al. 2010, MNRAS, 407, 2166 (G10)

Gandhi, P., Makishima, K., Durant, M., et al. 2008, MNRAS, 390, L29

Heinz, S., \& Sunyaev, R. A. 2003, MNRAS, 343, L59

Hynes, R. I., Steeghs, D., Casares, J., Charles, P. A., \& O’Brien, K. 2003, ApJ, 583, L95

Jarrett, T. H., Cohen, M., Masci, F., et al. 2011, ApJ, 735, 112

Kellermann, K. I., \& Pauliny-Toth, I. I. K. 1969, ApJ, 155, L71

Körding, E. G., Fender, R. P., \& Migliari, S. 2006, MNRAS, 369, 1451

Lutz, D., Feuchtgruber, H., Genzel, R., et al. 1996, A\&A, 315, L269

Mainzer, A., Bauer, J., Grav, T., et al. 2011, ApJ, 731, 53

Markoff, S., Falcke, H., \& Fender, R. 2001, A\&A, 372, L25

Markoff, S., Nowak, M., Corbel, S., Fender, R., \& Falcke, H. 2003, A\&A, 397, 645

Meier, D. L., Koide, S., \& Uchida, Y. 2001, Science, 291, 84

Migliari, S., Tomsick, J. A., Markoff, S., et al. 2007, ApJ, 670, 610

Migliari, S., Tomsick, J. A., Miller-Jones, J. C. A., et al. 2010, ApJ, 710, 117

Motch, C., Ilovaisky, S. A., \& Chevalier, C. 1982, A\&A, 109, L1

Muñoz-Darias, T., Casares, J., \& Martínez-Pais, I. G. 2008, MNRAS, 385, 2205

Pe'er, A., \& Casella, P. 2009, ApJ, 699, 1919

Polko, P., Meier, D. L., \& Markoff, S. 2010, ApJ, 723, 1343

Rahoui, F., Chaty, S., Rodriguez, J., et al. 2010, ApJ, 715, 1191

Rahoui, F., Lee, J. C., Heinz, S., et al. 2011, ApJ, 736, 63

Russell, D. M., Fender, R. P., Hynes, R. I., et al. 2006, MNRAS, 371, 1334

Rybicki, G. B., \& Lightman, A. P. 1979, Radiative Processes in Astrophysics (New York: Wiley-Interscience), 393

Shahbaz, T., Fender, R., \& Charles, P. A. 2001, A\&A, 376, L17

Shidatsu, M., Ueda, Y., Tazaki, F., et al. 2011, PASJ, in press (arXiv:1105.3586)

Titarchuk, L. 1994, ApJ, 434, 570

Tomsick, J. A., Bailyn, C. D., Buxton, M. M., et al. 2004, BAAS, 36, 104.04, 1516

Trippe, S., Krips, M., Pietu, V., et al. 2011, A\&A, in press (arXiv:1107.5456)

Vaughan, S., Edelson, R., Warwick, R. S., \& Uttley, P. 2003, MNRAS, 345, 1271

Vrtilek, S. D. 2008, in AIP Conf. Proc. 1010, A Population Explosion: The Nature \& Evolution of X-ray Binaries in Diverse Environments, ed. R. M. Bandyopadhyay, S. Wachter, D. Gelino, \& C. R. Gelino (Melville, NY: AIP), 18

Wachter, S. 2008, in AIP Conf. Proc. 1010, A Population Explosion: The Nature \& Evolution of X-ray Binaries in Diverse Environments, ed. R. M. Bandyopadhyay, S. Wachter, D. Gelino, \& C. R. Gelino (Melville, NY: AIP), 210

Wright, E. L., Eisenhardt, P. R. M., Mainzer, A. K., et al. 2010, AJ, 140, 1868 (W10) 